\title{
DETERMINANTAL VARIETIES, MONOMIAL SEMIGROUPS, AND ALGEBRAS ASSOCIATED WITH IDEALS ${ }^{1}$
}

\author{
JACOB BARSHAY
}

\begin{abstract}
This paper is concerned with the Rees and symmetric algebras of powers of ideals generated by $A$-sequences. These algebras are represented as quotients of polynomial rings over $A$ by ideals defined by minors of matrices. Their Krull dimensions are computed when $\boldsymbol{A}$ is a finite domain over a field. When $\boldsymbol{A}$ is a polynomial ring over a Cohen-Macaulay ring $A_{0}$ and the $A$ sequence consists of indeterminates, the Rees algebra is shown to be Cohen-Macaulay. If furthermore $A_{0}$ is a finite domain over a field, the symmetric algebra is shown to be Cohen-Macaulay only for $A$-sequences and squares of $A$-sequences of length two. Connections with algebras generated by monomials and Veronese varieties are pointed out.
\end{abstract}

1. Introduction. Let $A$ be a commutative ring with unit and $\boldsymbol{b}=$ $\left(b_{1}, \cdots, b_{r}\right)$ an ideal of $A$. Denote by $S(\boldsymbol{b})$ the symmetric algebra of $\boldsymbol{b}$ and by $R(b)$ the Rees algebra of $b$. Thus $R(b)=A\left[b_{1} T, \cdots, b_{r} T\right] \subseteq A[T]$. These graded algebras can be represented as quotients of polynomial rings via $\varphi: A\left[X_{1}, \cdots, X_{r}\right] \rightarrow S(b)$ given by $\varphi\left(X_{i}\right)=b_{i}$ and $\gamma: A\left[X_{1}, \cdots, X_{r}\right] \rightarrow$ $R(b)$ given by $\gamma\left(X_{i}\right)=b_{i} T$. Setting $Q=\operatorname{Ker} \varphi$ and $Q_{\infty}=\operatorname{Ker} \gamma$, we have that $Q$ is generated by all linear forms $\sum c_{i} X_{i}$ such that $\sum c_{i} b_{i}=0$ and $Q_{\infty}$ is generated by all forms $F\left(X_{1}, \cdots, X_{r}\right)$ such that $F\left(b_{1}, \cdots, b_{r}\right)=0$. We are interested in this paper in the case where $\boldsymbol{b}=\boldsymbol{a}^{n}, \boldsymbol{a}=\left(a_{1}, \cdots, a_{s}\right)$ $a_{1}, \cdots, a_{s}$ an $A$-sequence, $n \geqq 1$. In this situation, $Q$ and $Q_{\infty}$ are ideals defined by minors of matrices whose heights we compute when $A$ is a finite integral domain over a field. In the case where $A$ is a polynomial ring over a Cohen-Macaulay ring $A_{0}$ and $a_{1}, \cdots, a_{s}$ a subset of the variables, it is shown that $R\left(a^{n}\right)$ is always Cohen-Macaulay whereas $S\left(a^{n}\right)$ is Cohen-Macaulay only when $s=1$ or $n=1$ or $s=n=2$. The proof of the first employs Hochster's notion of a normal semigroup of monomials.

Received by the editors June 8, 1972 .

AMS (MOS) subject classifications (1970). Primary 13H10, 14M05, 15A78; Secondary 13B20, 13C15, 20M25.

Key words and phrases. Rees algebra, symmetric algebra, Cohen-Macaulay ring, perfect ideal, determinantal ideal, normal monomial semigroup, Veronese variety.

${ }^{1}$ This article was written while the author was supported by the Consiglio Nazionale delle Ricerche as Visiting Professor at the University of Genoa.

(c) American Mathematical Society 1973 
For the symmetric algebra we assume also that $A_{0}$ is a finite integral domain over a field. In the final section, we exhibit some connections between these graded algebras and Veronese varieties, reestablishing some properties of these varieties due to Gröbner.

2. Determinantal ideals and dimension of $R\left(a^{n}\right)$ and $S\left(a^{n}\right)$. Let $a_{1}, \cdots, a_{s}$ be an $A$-sequence, $a=\left(a_{1}, \cdots, a_{s}\right), n \geqq 1$. We want to compute the Krull dimension of $R\left(a^{n}\right)$ and $S\left(a^{n}\right)$ and determine when these rings are Cohen-Macaulay. The starting point for our considerations is the following theorem stating that $Q$ and $Q_{\infty}$ are determinantal ideals.

THEOREM 1. Let $a_{1}, \cdots, a_{s}$ be an A-sequence, $a=\left(a_{1}, \cdots, a_{s}\right), n \geqq 1$. Set $B=A\left[X_{I}\right]$ where $X_{I}=\left\{X_{i_{1} \cdots i_{s}} \mid \sum i_{\alpha}=n, i_{\alpha} \geqq 0\right\}$, a set of indeterminates of cardinality $\left(\begin{array}{c}n+s-1 \\ n\end{array}\right)$. Define a $\left[1+\left(\begin{array}{c}n+s-2 \\ n-1\end{array}\right)\right] \times s$ matrix $U$, the bottom $\left(\begin{array}{c}n+s-2 \\ n-1\end{array}\right)$ rows of which are indexed by $J=\left\{\left(j_{1}, \cdots, j_{s}\right) \mid \sum j_{\alpha}=n-1, j_{\alpha} \geqq 0\right\}$ as follows: $U_{1, k}=a_{k}$ and $U_{\left(j_{1}, \cdots, j_{s}\right), k}=X_{j_{1}, \cdots, j_{k-1}, j_{k}+1, j_{k+1}, \cdots, j_{s}}$. Then $R\left(a^{n}\right) \approx$ $B / Q_{\infty}$ and $S\left(a^{n}\right) \approx B / Q$ where $Q_{\infty}$ is the ideal generated by all $2 \times 2$ minors of $U$ and $Q$ is the ideal generated by those $2 \times 2$ minors of $U$ involving the first row.

Proof. [1, Theorems 1 and 4].

COROLlaRY 1. If $s=1$, then $R\left(a^{n}\right) \approx S\left(a^{n}\right) \approx A[X]$. Thus $\operatorname{dim} R\left(a^{n}\right)=$ $\operatorname{dim} S\left(\boldsymbol{a}^{n}\right)=1+\operatorname{dim} A$ and each is Cohen-Macaulay if $A$ is Cohen-Macaulay.

Corollary 2. If $n=1$, then $R(a) \approx S(a)$. If $A$ is Cohen-Macaulay, then each is Cohen-Macaulay of dimension $1+\operatorname{dim} A$.

Proof. $U$ is the $2 \times s$ matrix with first row $\left(a_{1}, \cdots, a_{s}\right)$ and second row $\left(X_{1}, \cdots, X_{s}\right)$. Thus $Q=Q_{\infty}$ and $R(a) \approx S(a)$. It is not hard to show [1, Proposition 2] that $Q$ is a perfect ideal of grade $s-1$. Thus $S(a)$ is Cohen-Macaulay if $A$ is and $\operatorname{dim} S(a)=\operatorname{dim} B-$ ht $Q=s+\operatorname{dim} A-\operatorname{gr} Q=$ $1+\operatorname{dim} A$.

We can assume henceforth that $s, n \geqq 2$.

Proposition 1. Notation as in Theorem 1. Then $Q_{\infty} \cdot a B \subseteq Q \subseteq Q_{\infty} \cap a B$.

Proof. Let $F=U_{(j), k} U_{\left(j^{\prime}\right), k^{\prime}}-U_{\left(j^{\prime}\right), k} U_{(j), k^{\prime}}$ be a generator of $Q_{\infty}$ not among the generators of $Q$. We must show that $a_{m} F \in Q$ for $m=1, \cdots, s$. Consider the matrix

$$
\left(\begin{array}{ccc}
a_{k} & a_{k^{\prime}} & a_{m} \\
U_{(j) . k} & U_{(j), k^{\prime}} & U_{(j), m} \\
U_{\left(j^{\prime}\right), k} & U_{\left(j^{\prime}\right), k^{\prime}} & U_{\left(j^{\prime}\right), m}
\end{array}\right) .
$$

Comparing the expressions for the determinant, first by expanding along 
the third row, then by expanding along the third column, we see that $a_{m} F \in Q$. Thus $Q_{\infty} \cdot a B \subseteq Q$. The other inequality is obvious.

Corollary. ht $Q=\min \left(\right.$ ht $\left.Q_{\infty}, s\right)$.

Proof. Since $a$ is generated by an $A$-sequence of length $s$, ht $a B=s$. In general for any ideals $I$ and $J$ in a Noetherian ring, ht $(I \cap J)=h t(I J)=$ $\min ($ ht $I$, ht $J$ ).

Thus if we know ht $Q_{\infty}$, we know ht $Q$. Furthermore we can compute ht $Q_{\infty}$ from $\operatorname{dim} R\left(a^{n}\right)$ and $\operatorname{dim} B$ provided that $B$ satisfies the chain condition ht $b+\operatorname{dim} b=\operatorname{dim} B$ for any ideal $b$ of $B$.

Proposition 2. Suppose that $A$ is a finite integral domain over a field $k$, $0 \neq b$ any ideal of $A$. Then $\operatorname{dim} R(b)=1+\operatorname{dim} A$.

Proof. Let $K$ be the fraction field of $A$ and $b=\left(b_{1}, \cdots, b_{r}\right)$. Then $R(b)=A\left[b_{1} T, \cdots, b_{r} T\right] \subseteq A[T]$. The fraction field of $R(b)$ contains $K$ and $T=b_{1}^{-1}\left(b_{1} T\right)$ and so equals $K(T)$. Thus $\operatorname{dim} R(b)=t d_{k}(K(T))=1+t d_{k}(K)=$ $1+\operatorname{dim} A$.

COROLlary 1. Notation as in Theorem 1 with $A$ a finite integral domain over a field. Then ht $Q_{\infty}=\left(\begin{array}{c}n+s-1 \\ n\end{array}\right)-1$.

Proof. $\operatorname{dim} B=\operatorname{dim} R\left(a^{n}\right)+$ ht $Q^{\infty}$ where $\operatorname{dim} B=\operatorname{dim} A+\left({ }_{n}^{n+s-1}\right)$ and $\operatorname{dim} R\left(a^{n}\right)=\operatorname{dim} A+1$.

COROllary 2. Notation as in Theorem 1 with $A$ a finite integral domain over a field. Then (i) ht $Q=s$, (ii) $\operatorname{dim} S\left(a^{n}\right)=\left(\begin{array}{c}n+s-1 \\ n\end{array}\right)-s+\operatorname{dim} A$.

Proof. (i) By the corollary to Proposition 1, it suffices to observe that $\left({ }_{n}^{n+s-1}\right) \geqq s+1$ when $s, n \geqq 2$. In fact,

$$
(s-1) !\left(\begin{array}{c}
n+s-1 \\
n
\end{array}\right) \geqq \frac{(s+1) !}{2} \geqq(s-1) !(s+1)
$$

where the first inequality is strict unless $n=2$ and the second inequality is strict unless $s=2$.

$$
\operatorname{dim} S\left(a^{n}\right)=\operatorname{dim} B-\text { ht } Q=\left(\begin{array}{c}
n+s-1 \\
n
\end{array}\right)+\operatorname{dim} A-s .
$$

3. Monomial semigroups and Cohen-Macaulay properties. We now turn to the question of when $R\left(a^{n}\right)$ or $S\left(a^{n}\right)$ is Cohen-Macaulay, considering only the special case $A=A_{0}\left[T_{1}, \cdots, T_{s}, T_{s+1}, \cdots, T_{r}\right], a=$ $\left(T_{1}, \cdots, T_{s}\right), A_{0}$ a ring.

Proposition 3. Let $A=A_{0}\left[T_{1}, \cdots, T_{s}, T_{s+1}, \cdots, T_{r}\right], a=\left(T_{1}, \cdots, T_{s}\right)$, $R\left(a^{n}\right) \approx B / Q_{\infty}, S\left(a^{n}\right) \approx B / Q$ where $B=A\left[X_{I}\right]$ is a polynomial ring over $A$ in $\left(\begin{array}{c}n+s-1 \\ n\end{array}\right)$ indeterminates. Then $Q=Q_{\infty} \cap a B$. 
Proof. It suffices by Proposition 1 to show that $Q_{\infty} \cap a B \subseteq Q$. Set $B=B_{0}\left[T_{1}, \cdots, T_{s}\right]$ where $B_{0}=A_{0}\left[T_{s+1}, \cdots, T_{r}, X_{I}\right]$. By Theorem 1, $Q_{\infty}=\left(b_{1}, \cdots, b_{p}, c_{1}, \cdots, c_{q}\right)$ where $b_{i} \in B_{0}$ and $\left(c_{1}, \cdots, c_{q}\right)=Q \subseteq a B$. If $x \in Q_{\infty} \cap a B$, then $x=\sum f_{i} b_{i}+y$ where $f_{i} \in B, y \in Q \subseteq a B$.

Writing $f_{i}=g_{i}+h_{i}$ where $g_{i} \in B_{0}, h_{i} \in a B$, we have $x=\sum g_{i} b_{i}+$ $\sum h_{i} b_{i}+y$ where $\sum h_{i} b_{i} \in Q_{\infty} \cdot a B \subseteq Q$ by Proposition 1. Thus

and so $x \in Q$.

$$
\sum g_{i} b_{i} \in B_{0} \cap a B=(0)
$$

Theorem 2. Notation as in Proposition 3. Suppose that $A_{0}$ is a finite integral domain over a field and Cohen-Macaulay. Then $S\left(a^{n}\right)$ is CohenMacaulay if and only if $s=n=2$.

Proof. If $S\left(a^{n}\right)$ is Cohen-Macaulay, then $Q$ must be an unmixed ideal of $B$. The previous proposition shows that $Q$ is the intersection of two prime ideals whose heights are different unless $s=n=2$ (Corollary 2, Proposition 2).

Conversely, if $s=n=2$, then $B=A\left[X_{1}, X_{2}, X_{3}\right]$ and $Q=\left(T_{1} X_{2}-T_{2} X_{1}\right.$, $\left.T_{1} X_{3}-T_{2} X_{2}\right)$. The generators of $Q$ clearly form a $B$-sequence. Hence $Q$ is a perfect ideal. Since $B$ is Cohen-Macaulay, it follows that $S\left(\left(T_{1}, T_{2}\right)^{2}\right)$ is Cohen-Macaulay.

The notion of a normal semigroup of monomials introduced by Hochster turns out to be very useful in looking at the Rees algebra. The next proposition and theorem give equivalent definitions of this concept and the key property of adjoining such a semigroup to a Cohen-Macaulay ring.

A monomial in the indeterminates $X_{1}, \cdots, X_{n}$ is a term of the form $X_{1}^{m_{1}} X_{2}^{m_{2}} \cdots X_{n}^{m_{n}}$ where $m_{i} \geqq 0$. A semigroup of monomials $M$ is a set of monomials closed under product and containing the monomial $1=$ $X_{1}^{0} \cdots X_{n}^{0}$. If $R$ is any ring over which $X_{1}, \cdots, X_{n}$ are algebraically independent, we can consider the semigroup ring $R[M] \subseteq R\left[X_{1}, \cdots, X_{n}\right]$. An integral domain is called normal if it is Noetherian and integrally closed.

Proposition 4. Let $M$ be a monomial semigroup in $X_{1}, \cdots, X_{n}$. The following conditions are equivalent:

(i) $M$ is finitely generated and for any $p, q, r \in M$ and any integer $m \geqq 1$ satisfying $p q^{m}=r^{m}$, there exists $t \in M$ such that $p=t^{m}$.

(ii) There exists a field $K$ such that $K[M] \subseteq K\left[X_{1}, \cdots, X_{n}\right]$ is normal.

(iii) $M$ is finitely generated and for any integrally closed domain $D$, $D[M] \sim\left[X_{1}, \cdots, X_{n}\right]$ is integrally closed.

Proof. [6, Proposition 1]. 
Definition. A monomial semigroup satisfying the conditions of Proposition 4 is called normal.

EXAMPLE. Let $M_{s, n}$ be the semigroup generated by all monomials of degree $n$ in $X_{1}, \cdots, X_{s}$. Then $M_{s, n}$ is finitely generated and is easily seen to satisfy condition (i) by taking $t=r q^{-1}$. It is in fact what Hochster calls a full ( $\Rightarrow$ normal) semigroup.

THEOREM 3. If $R$ is a Cohen-Macaulay ring and $M$ is a normal monomial semigroup, then $R[M]$ is Cohen-Macaulay.

Proof. [6, Theorem 1].

In applying these ideas, we need the following fact whose proof can be found in [1, Corollary 2, Theorem 5].

Proposition 5. Let $A_{0}$ be an integrally closed domain,

$$
A=A_{0}\left[T_{1}, \cdots, T_{s}, T_{s+1}, \cdots, T_{r}\right],
$$

$a=\left(T_{1}, \cdots, T_{s}\right), n \geqq 1$. Then $R\left(a^{n}\right)$ is integrally closed

Combining the last three results, we obtain

Theorem 4. Let $A_{0}$ be a Cohen-Macaulay ring, $A=A_{0}\left[T_{1}, \cdots, T_{s}\right.$, $\left.T_{s+1}, \cdots, T_{r}\right], a=\left(T_{1}, \cdots, T_{s}\right), n \geqq 1$. Then $R\left(a^{n}\right)$ is Cohen-Macaulay.

Proof. Let $M$ be the monomial semigroup generated by $T_{1}, \cdots, T_{r}$ and all monomials $m T$ where $m$ is a monomial of degree $n$ in $T_{1}, \cdots, T_{s}$. Then $R\left(\grave{a}^{n}\right) \approx A_{0}[M] \subseteq A_{0}\left[T_{1}, \cdots, T_{r}, T\right]$. By Proposition $5, A_{0}[M]$ is an integrally closed-domain if $A_{0}$ is an integrally closed domain. Since $M$ is finitely generated, $M$ is normal by Proposition 4 . Then by Theorem 3, $\boldsymbol{R}\left(\boldsymbol{a}^{n}\right)$ is Cohen-Macaulay.

\section{Veronese varieties and generic, symmetric matrices.}

Definition. The Veronese variety $V_{s, n}$ over a field $k$ is the projective variety whose homogeneous coordinate ring is generated over $k$ by all monomials of degree $n$ in the indeterminates $T_{1}, \cdots, T_{s}$, or, in our previous notation, $k\left[M_{s, n}\right]$.

Introducing $\left(\begin{array}{c}n+s-1 \\ n\end{array}\right)$ indeterminates $X_{I}=\left\{X_{i_{1} \cdots i_{s}} \mid \sum i_{\alpha}=n, i_{\alpha} \geqq 0\right\}$, let $I_{s, n}$ denote the kernel of the map $k\left[X_{I}\right] \rightarrow k\left[M_{s, n}\right]$ given by $X_{i_{1} \cdots i_{s}} \mapsto T_{1}^{i_{1}} \cdots T_{s}^{i_{s}}$. $I_{s, n}$ is generated by all forms $F\left(X_{I}\right)$ such that $F\left(T^{I}\right)=0$. In fact $I_{s, n}$ is generated by quadratic forms. More precisely, if we denote by $U^{\prime}$ the matrix obtained from the matrix $U$ of Theorem 1 by removing the first row, then $I_{s, n}$ is generated by all $2 \times 2$ minors of $U^{\prime}$ (see [3], [4]). The normality of the monomial semigroup $M_{s, n}$ thus gives

THEOREM 5. The coordinate ring of the Veronese variety $V_{s, n}$ is arithmetically normal and Cohen-Macaulay. 
REMARK. The "Cohen-Macaulay" part of the above theorem was proved by Gröbner [5] who showed by direct methods that $I_{s, n}$ is perfect.

Taking $n=2$, the matrix $U^{\prime}$ becomes a generic, symmetric $s \times s$ matrix. Thus we have the following

COROLlaRY. Let $X$ be a generic, symmetric $s \times s$ matrix, that is, the matrix whose $(i, j)$ and $(j, i)$ entries are $X_{i j}(1 \leqq i \leqq j \leqq s)$. Denote by I the ideal generated by all $t \times t$ minors of $X$ in the polynomial ring $k\left[X_{i j}\right]$. Then $I_{t}$ is perfect of grade $\left({ }_{2}^{s+2-t}\right)$ for $t=1,2$, or $s$.

Proof. The result is trivial for $t=1$ or $s$. For $t=2, I_{2}$ is just the defining ideal of $V_{s, 2}$ which is Cohen-Macaulay. Hence $I_{2}$ is perfect. Furthermore

$$
\text { gr } I_{2}=\text { ht } I_{2}=\operatorname{dim} k\left[X_{i j}\right]-\operatorname{dim} k\left[M_{s, 2}\right]=\left(\begin{array}{c}
s+1 \\
2
\end{array}\right)-s=\left(\begin{array}{l}
s \\
2
\end{array}\right) .
$$

Note that $\operatorname{dim} k\left[M_{s, 2}\right]=s$ since $k\left[M_{s, 2}\right]$ is an integral extension of $k\left[X_{1}^{2}, \cdots, X_{s}^{2}\right]$.

It seems reasonable to conjecture that the above corollary is true for all values of $t .^{2}$ It is also interesting to compare this fact with the recent theorem of Hochster and Eagon ([7], [8]) that the grade of the ideal generated by $t \times t$ minors of an $r \times s$ matrix is bounded above by $(r-t+1)(s-t+1)$ and if equality holds, the ideal is perfect. This was previously known for $t=\min (r, s)$ [2] and $t=2$ ([12], [13]). For the generic, symmetric $s \times s$ matrix, $I_{2}$ is perfect of grade $\left(\begin{array}{l}s \\ 2\end{array}\right)<(r-t+1) \times$ $(s-t+1)=(s-1)^{2}$ for $s \geqq 3$. Perhaps the corresponding theorem holds for symmetric $s \times s$ matrices with the upper bound $\left({ }_{2}^{s+2-t}\right)$.

\section{BIBLIOGRAPHY}

1. J. Barshay, Graded algebras of powers of ideals generated by A-sequences, J J. Algebra 25 (1973), 90-99.

2. J. A. Eagon and D. G. Northcott, Ideals defined by minors of matrices and a certain complex associated with them, Proc. Roy. Soc. Ser. A 269 (1962), 188-204. MR $26 \# 161$.

3. L. S. Goddard, Bases for the prime ideals associated with certain classes of algebraic varieties, Proc. Cambridge Philos. Soc. 39 (1943), 35-48. MR 4, 168.

4. — Prime ideals and postulation formulae, Proc. Cambridge Philos. Soc. 44 (1948), 43-49. MR 9, 304.

5. W. Gröbner, Ubber Veronesesche Varietäten und deren Projektionen, Arch. Math. 16 (1965), 257-264. MR 32 \#1191.

6. M. Hochster, Rings of invariants of tori, Cohen-Macaulay rings generated by monomials, and polytopes, Ann. of Math. (2) 96 (1972), 318-337. [14].

2 The referee has informed me that this conjecture has been settled by $R$. Kutz 
7. M. Hochster and J. A. Eagon, A class of perfect determinantal ideals, Bull. Amer. Math. Soc. 76 (1970), 1026-1029. MR 42 \#1814.

8. - Cohen-Macaulay rings, invariant theory, and the generic perfection of determinantal loci, Amer. J. Math. 93 (1971), 1020-1058.

9. D. G. Northcott, Semi-regular rings and semi-regular ideals, Quart. J. Math. Oxford Ser. (2) 11 (1960), 81-104. MR 22 \#5653.

10. - Some remarks on the theory of ideals defined by matrices, Quart. J. Math. Oxford Ser. (2) 14 (1963), 193-204. MR 27 \#1467.

11. D. Rees, The grade of an ideal or module, Proc. Cambridge Philos. Soc. 53 (1957), 28-42. MR 18, 637.

12. D. W. Sharpe, On certain polynomial ideals defined by matrices, Quart. J. Math. Oxford Ser. (2) 15 (1964), 155-175. MR 29 \#1226.

13. - The syzygies and semi-regularity of certain ideals defined by matrices, Proc. London Math. Soc. (3) 15 (1965), 645-679. MR 32 \#5684.

14. R. Kutz, Cohen-Macaulay rings and ideal theory in rings of invariants of algebraic groups, Thesis, University of Minnesota, Minneapolis, Minn., 1972.

Department of Mathematics, City College, City University of New York, NeW YoRK, New YORK 10031 